\title{
Development of a Great Britain Transmission System Reduced Model for Hardware-In-the-Loop Studies
}

\author{
Peter Imris, Martin Bradley, Gareth Taylor \\ Department of Electronic and Computer Engineering \\ Brunel University London \\ Uxbridge, UB8 3PH, UK \\ Peter.Imris@brunel.ac.uk, \\ Martin.Bradley@brunel.ac.uk, \\ Gareth.Taylor@brunel.ac.uk
}

\author{
Yun Li \\ National Grid Electricity System Operator \\ St. Catherine's Lodge \\ Wokingham, RG41 5BN, UK \\ Yun.Li@nationalgrideso.com
}

\begin{abstract}
This paper presents the development of a reduced model of the Great Britain (GB) transmission system for Hardware-In-the-Loop (HIL) studies. The original reduced model of the GB system was designed for dynamic simulations using the power system software DIgSILENT PowerFactory. In this paper a reduced model is developed for real-time simulation and enhancements to the original DIgSILENT PowerFactory models have been implemented in order to use it with the real-time dynamic simulation tool of OPAL-RT Technologies, ePHASORSIM. In this paper it is demonstrated that the implemented enhancements do not have an adverse impact on model accuracy or efficacy. It is important to note that the developed model can be directly interfaced with the OPAL-RT real-time digital simulator for HIL studies. It is also important to note that the modelling interface methodology that is presented with regard to the development of the reduced model of the GB system is also applicable to a full system model of the GB transmission system.
\end{abstract}

Index Terms-- Loss of Mains protection, Hardware-In-theLoop, Real-time digital simulation, Reduced model.

\section{INTRODUCTION}

The composition of electricity generation has changed significantly in recent years across the world [1]. This is mainly due to the increasing amounts of renewable energy sources, such as wind and solar, that are being connected to power systems in parallel with the decommissioning of conventional power stations, such as coal, for environmental reasons and the sustainability of the power production. This trend is expected to continue in coming years in order to meet environmental targets and legislation.

The Great Britain (GB) power system currently experiences a high penetration of the renewables. The increasing rate of renewable penetration into the power system, however, brings new challenges previously not faced. The renewable generation (mainly solar or wind) is

The research was sponsored by National Grid ESO with Network Innovation Allowance funding from OFGEM (Office of Gas and Electricity Markets). connected to the power system via grid-following electronic power convertors and thus does not provide any inertial response or resistance to vector shifts. The system inertia is also decreasing as a result of the installation of new HVDC links in the GB power system, which are also displacing synchronous machines that traditionally provided the inertia. A number of solutions are proposed in the literature to substitute the lack of physical inertia from renewables and HVDC links, for example by the so-called artificial, emulated, simulated or synthetic inertia or Virtual Synchronous machines (VSM) and Grid Forming Convertors (GFC), [2] [3] [4] [5], [6], but these new concepts have not been yet widely applied to power systems. In addition, the total inertia of the power system is represented by the sum of the inertia of all the synchronous generators as well as the residual inertia of the demand. In recent years not only has the inertia of the generation mix been changing but also the inertia of the demand. Overall, the total inertia of the GB transmission system has decreased significantly over the past 20 years as a consequence of the reduction in rotating synchronous generators and changes in demand [7], [8] as shown Figures 1 and 2.

The decrease of system inertia influences the dynamic behaviour of the whole GB system. For instance, disturbances in the power system such as generator or interconnector tripping can lead to larger frequency fluctuations across wider geographical areas, particularly a larger Rate of Change of Frequency (RoCoF), when compared to historical situations where system inertia was higher. If unmitigated, this fluctuation may in some cases exhibit similar behaviour to that which is present during system islanding and thus may lead to issues including the maloperation of Loss-of-Mains (LoM) protection relays as well as cascade tripping of distributed generation [1]. It should be noted that mal-operation in this context refers to operation when islanding has not occurred. This additional loss of the power will increase the impact of the original disturbance on the system. In order to manage this risk for secured system events, NGESO takes a range of measures 
including managing the size of the largest import/export loss and the minimum system inertia. Securing the RoCoF risk has been costing approximately $£ 15 \mathrm{~m}$ per month, and this may increase with further growth in renewables on the system, [9]. NGESO recently agreed contracts with five parties, worth $£ 328$ million over a six-year period, to provide 12.5 GVA seconds of inertia [10]. There is also an industrywide initiative to change the settings on LoM protection relays to make them less sensitive. During 2018 the Energy Networks Association (ENA) in the UK proposed updates to Engineering Recommendation G59 for LoM settings. A new standard G99 was introduced for LoM relay settings for new generator installations above $50 \mathrm{MW}$ [11]. G99 amended the RoCoF threshold from $0.125 \mathrm{~Hz} / \mathrm{s}$ to $1 \mathrm{~Hz} / \mathrm{s}$ and prohibited the use of Vector Shift protection. In addition to this, an ENA programme to update existing LoM relays to the new standard is also underway and is due to complete by August 2022 [12], but it does not cover legacy small-scale installations which may continue to present a risk of tripping.

The unwanted disconnection of renewables is not just a theoretical possibility, but also a real experience in the GB power system. For example, on 9 August 2019 a lightning strike led to the near-simultaneous loss of two independent generators totalling $981 \mathrm{MW}$ together with approximately $150 \mathrm{MW}$ of embedded generation tripping due to Vector Shift LoM protection. The combined total exceeded the secured infeed loss, and the fall in frequency led to a further tripping of $350 \mathrm{MW}$ of embedded generation due to RoCoF LoM protection. The frequency fell to $48.8 \mathrm{~Hz}$ which led to automatic disconnection of approximately 1 million customers by Low Frequency Demand Disconnection (LFDD) relays [13].

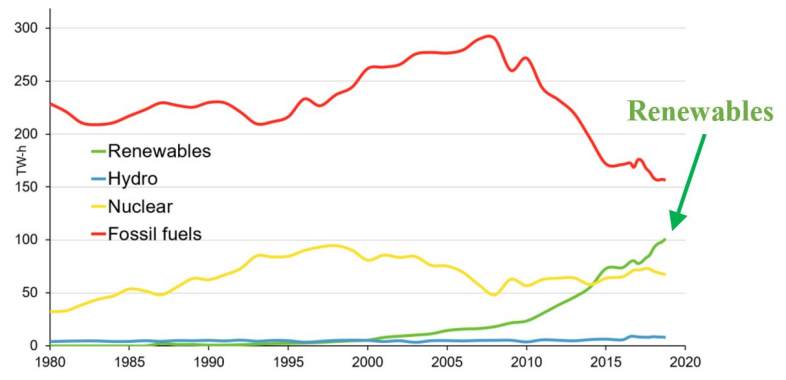

Figure 1 UK electricity production by source 1980-2018 [2].

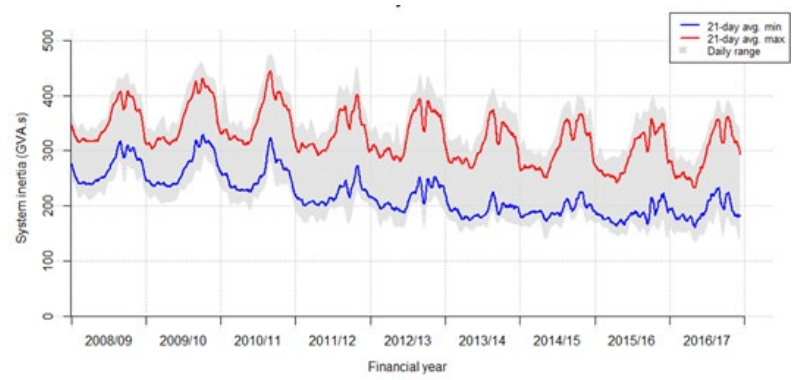

Figure 2 Historic system inertia of the GB system [3].

The fast frequency phenomena on the GB transmission system can be studied by analysing Phasor Measurement Unit (PMU) data from recorded disturbance events on the GB transmission system [14], [15]. It is important to note that PMUs are already installed across the GB system in key locations [16] and future plans are in place to install PMUs at every Grid Supply Point (GSP) [17]. However, the limited number of relevant disturbance events that occur at present gives rise to the need to investigate a wider range of potential events including Future Energy Scenarios [18], especially with regards to decreasing system inertia and related fault levels. Thereby, requiring the need for further research of frequency phenomena within an integrated real-time simulation environment. NGESO has developed two models of the GB transmission system implemented in DIgSILENT PowerFactory. They are a full system model [18] and a reduced model of GB transmission system [19]. These models were originally built for offline studies. However, the use of these PowerFactory models for real time computation requires further development in order to be used with the OPAL-RT dynamic simulator ePHASORSIM. These changes to the models are proposed and tested in this paper with the reduced model.

\section{REDUCED MODEL OF THE GB TRANSMISSION SYSTEM}

\section{A. Reduced model origins}

NGESO originally developed a full scale model of the GB transmission system in DIgSILENT PowerFactory, as an Offline Transmission Analysis tool (OLTA) [18]. This is a detailed model, which represents the actual GB transmission system including the topology, generation, power lines, and HVDC links such that the static and dynamic behaviour closely match the real system. However, this model used internally by NGESO is complex and not publicly available for confidentiality reasons. Therefore, National Grid also developed a reduced model in 2012. The use of nonconfidential data and its ability to quickly create various future scenarios makes it particularly useful for academic research [19], [20].

\section{B. Reduced model design}

The reduced model of the GB transmission system was derived from the full system model [19]. The full model of the GB transmission system consists of regions that are referred to as FLOP zones as described in Figure 3, [20]. Each zone of the reduced model is aligned to the full GB model but only has one substation per zone, which for simplicity has an identical structure with a mixture of various types of generation and load as presented in Figure 4. Each zone contains the following types of generation typically used in the UK: gas, coal, nuclear, pumped storage, oil, hydro, marine, biomass, wind, static generators and HVDC links. The generators of the zone model represent the aggregated generation of the actual zone. If a certain type of generation does not exist in the particular zone it is set to zero output. The total load of a zone is also aggregated and represented by one demand only. The power lines in the reduced model are virtual lines which may represent numerous circuits in the full model with the equivalent electrical distances between zones, Figure 3. The dynamic behaviour of the generators is modelled using non-standard user-defined controllers, namely Automatic Voltage 
Regulators (AVR), Governors (GOV) and - depending on generation type - Power System Stabilizers (PSS). These controllers were developed by National Grid to match the behaviour of each type of generation.

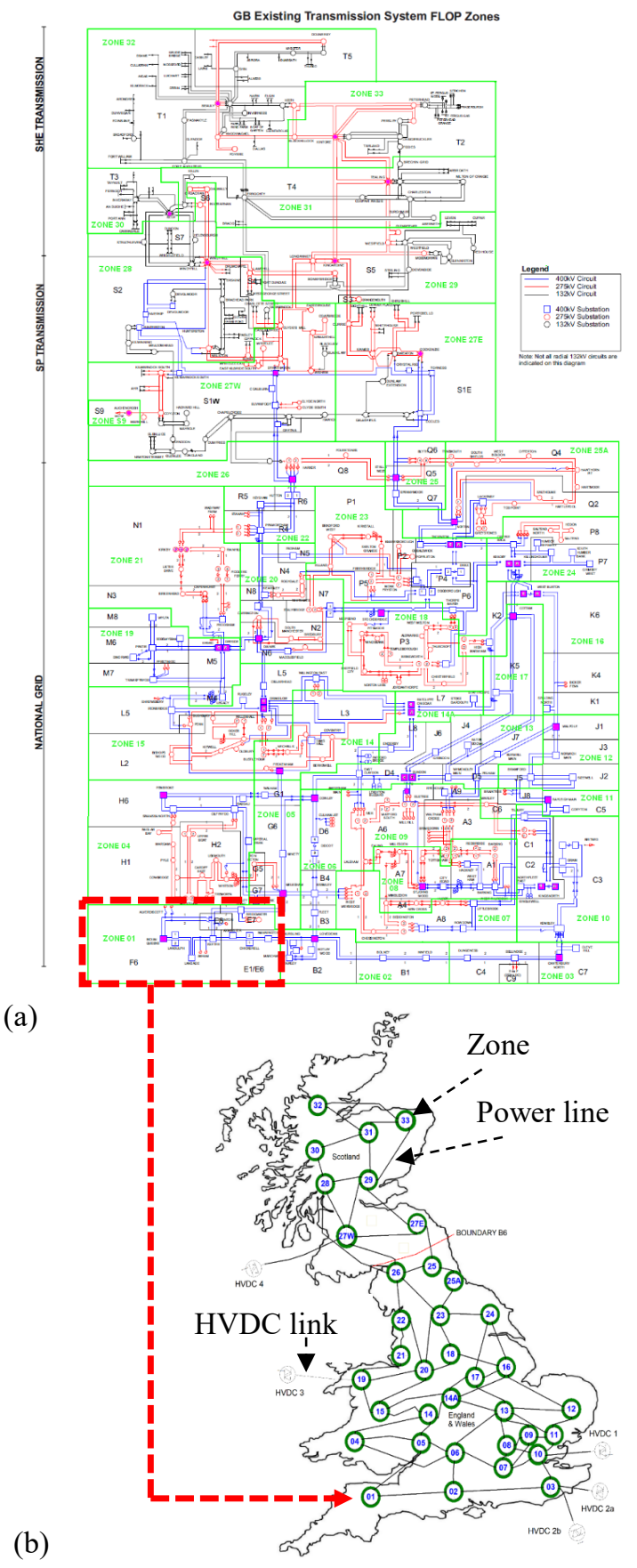

Figure 3 Creation of the reduced model from the full model of the GB transmission system. (a) Full model and (b) reduced model. The red arrow indicates Zone 01 as based on the FLOP zones, [20].

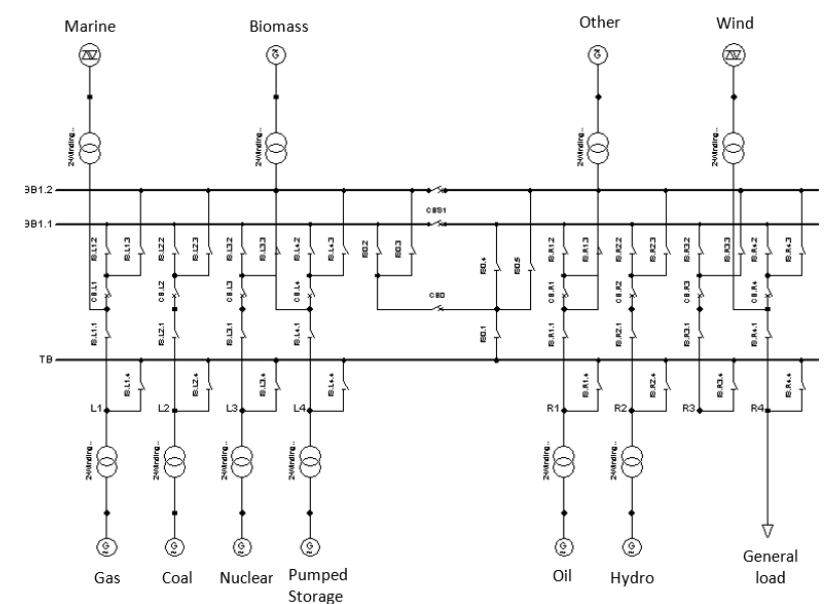

Figure 4 Structure of a single zone implemented in PowerFactory, [19]

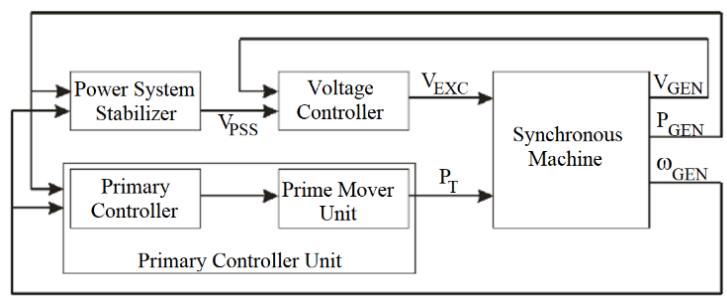

Figure 5 PowerFactory model for control block of synchronous generators.

A complex HVDC model, as presented in Figure 6, has been included in the reduced network to represent interconnectors to the GB network. It is based on the Line Commutated Converter (LCC) type HVDC.

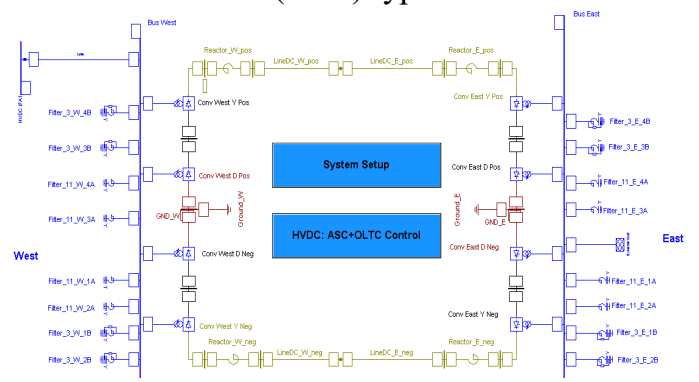

Figure 6 The HVDC link model based on a Line Commutated Converter used in the original reduced model in PowerFactory.

\section{PROposed Updates of THE Reduced Model}

\section{A. Compatibility of ePHASORSIM \& PowerFactory}

The real-time digital simulator OPAL-RT uses a phasor based transient stability simulator called ePHASORSIM for dynamic simulations [21]. The basic data entry tool in ePHASORSIM is an Excel Workbook; however, it also offers a straightforward way to import data from other simulation packages (PSS/e, CYME and PowerFactory). A model with the following components can be automatically imported from PowerFactory: bus; load; synchronous 
machine; machine controller; line; two-winding transformer; three-winding transformer; switch. OPAL-RT supports the most commonly used types of controllers (GOVs, AVRs and PSSs) [22]. However, the controllers of the reduced model are non-standard defined by NGESO and implemented in DSL scripts (DIgSILENT Simulation Language) [19], and thus an automatic conversion of the models to ePHASORSIM is not possible in the current version of OPAL-RT.

\section{B. Proposed enhancements to the reduced model}

In order to automatically convert the reduced model, as presented in Figure 3b with the zone structure as in Figure 4, from PowerFactory to ePHASORSIM and also to speed up the simulations the following changes were applied:

- Reduction in the number of nodes in a zone. (1) Reduction of multiple buses in each zone to one bus only. (2) Removing non-essential switches from the zone.

- $\quad$ Replacement of the AVRs, GOVs and PSSs with standard types supported by OPAL-RT. The userdefined controllers were substituted with the closest equivalent (IEEE type ST1 excitation system model - EXST1, Steam turbine-governor model - TGOV1 and Speed sensitive stabilizer model - STAB1) and the parameters were adjusted to provide a similar response to the original model, [20].

- Synchronous generators were updated to represent the following types of generation: nuclear, coal, pumped storage, hydro, CHP, biomass and gas. Oil generation was removed from the model as this generation type is no longer in service in GB. "Other" generation was also removed as all the significant generation types were included.

- Static generation: The complex HVDC LCC model was replaced by a simple static generator/load. Solar generation was added to the model in static form, so that static load/generation is used to represent HVDC links, solar and wind generation.

The single zone of the enhanced reduced model is shown in Figure 7.

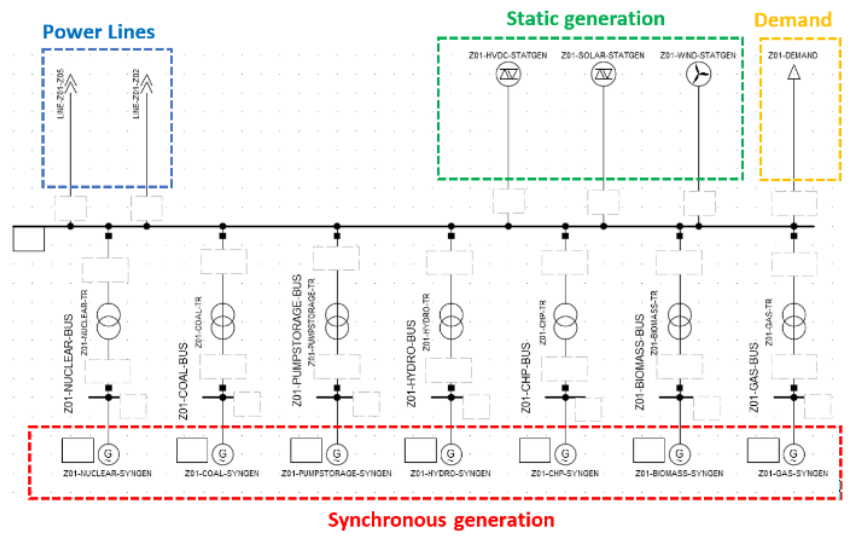

Figure 7 New structure of a single zone implemented in PowerFactory, [20].

\section{Simulations}

\section{A. GB system case study}

This GB system case study represents a real HVDC link trip in the South East of the transmission system, while importing $500 \mathrm{MW}$ to GB. The location of disturbance in the reduced model is Zone 03, as presented in Figure 3. The system conditions during the event are: total system demand 33.4 GW, inertia 248.7 GVAs and frequency excursion $49.99-49.70 \mathrm{~Hz},[20]$.

\section{B. Simulation of the case study}

The reduced model tested in this section was updated with the modified zonal generation and inertia parameters based on the real network conditions for the HVDC trip event. For modelling purposes, the generation, demand and inertia were aggregated for each zone based on data from the NGESO OLTA full system model at the moment of the disturbance event according to Equations (1), (2) and (3).

$$
\begin{aligned}
& S_{g n}=\sum_{i=1}^{N} S_{g n i}, \quad \text { [MVA] } \\
& H_{g n}=\frac{\sum_{i=1}^{n} H_{i} S_{g n i}}{\sum_{i=1}^{n} S_{g n i}}, \quad \text { [MVAs] } \\
& P_{a c t}=\sum_{i=1}^{N} P_{a c t ~}, \quad[\mathrm{MW}]
\end{aligned}
$$

Where:

$\begin{array}{ll}N & =\text { number of generators in zone for the same type; } \\ S_{g n} & =\text { rating of the generator (MVA); } \\ H_{g n} & =\text { inertia constant of the generator }(\mathrm{s}) ; \\ P_{a c t} & =\text { actual power of the generator (MW); } \\ i & =\text { suffix for the computation. }\end{array}$

A technique presented in [23] was applied to fine tune the model response for the standard governor TGOV1 model, as shown in Figure 8. By adjusting the TGOV1 parameters a similar response was achieved. Detailed parameters of the 
generator's controller settings (AVRs GOVs and PSSs) are presented in [20].

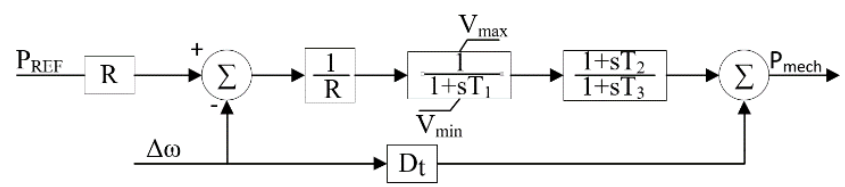

Figure 8 Block diagram of turbine governor [23]

The disturbance was applied to the model in Zone 03 and the results compared at selected points with the PMU data, for example Zone 17 with the PMU data from the Staythorpe substation during the actual event. The case study was simulated with DIgSILENT PowerFactory and OPAL-RT ePHASORSIM. The validation below is focused on the first few seconds after the disturbance as this time is critical for the LoM relay operation. The response of the reduced model is compared to the PMU data for the selected substations in Figure 9.

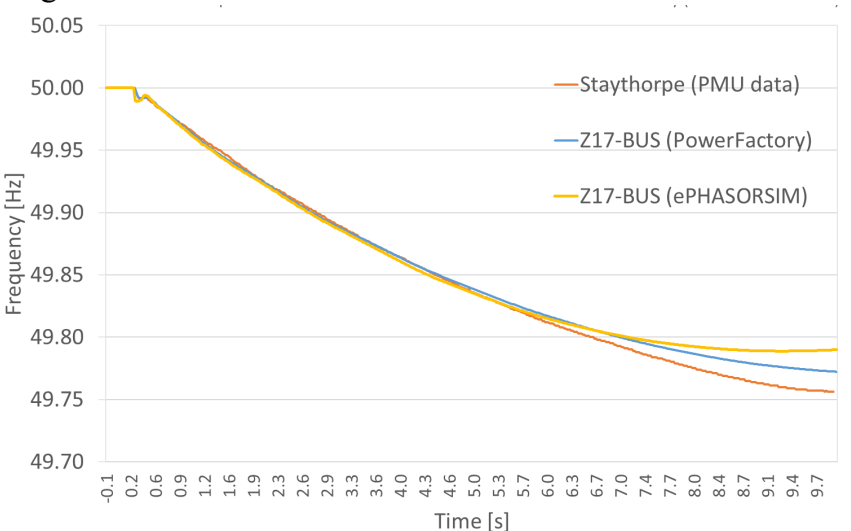

Figure 9 Simulation results using an enhanced reduced model in PowerFactory and ePHASORSIM as compared with PMU data from an actual disturbance event.

\section{ANALYSIS OF RESULTS}

As seen from the results which are presented in Figure 9, the proposed enhancements to the model have only a slight impact on the model accuracy. Test results from PowerFactory (the enhanced model as described in Section III) and ePHASORSIM show that very good accuracy was achieved for the first 5 seconds of the disturbance, then the results differ slightly. The initial change in frequency in the model predominantly relates to the system inertia, which was the same in all the test cases. The main purpose of this modelling is to test the LoM protection relays in HIL, and these relays would react to the initial frequency change; therefore, despite the differences in results at later times, the models can be considered as sufficiently accurate for the period of interest.

\section{CONCLUSIONS AND FUTURE RESEARCH}

This paper presented the development of a reduced model of the GB transmission system for HIL studies. The proposed changes to the model enable an automatic conversion of the reduced model as developed in DIgSILENT PowerFactory to a real-time simulation platform based on OPAL-RT ePHASORSIM. The main changes in the model are the replacement of the nonstandard user-defined controllers (AVRs, GOVs, PSSs) with the components supported by OPAL-RT, tuning the controller parameters, and substituting a complex HVDC link model with a simple static load/generator. The changes to the number of nodes (topology) of an individual zone contributes to the reduction in computational power needed for real-time simulation. In producing this paper, we have demonstrated that the PowerFactory model can be directly interfaced with the OPAL-RT real-time digital simulator for HIL studies. Based on the results presented here, the implemented changes to the reduced model do not have an adverse impact on model accuracy or efficacy. It is also important to note that the modelling interface methodology that is presented with regard to the development of the reduced model of the GB system is also applicable to a full system model of the GB transmission system. Future research will use HIL studies to investigate the impact of reduced system inertia on RoCoF LoM protection for distributed generation.

\section{ACKNOWLEDGMENT}

The research was sponsored by NGESO with Network Innovation Allowance funding from OFGEM (Office of Gas and Electricity Markets).

The authors also thank Richard Ierna of NGESO and Vahid Jalili-Marandi from OPAL-RT for their contributions and valuable comments in relation to this paper.

\section{REFERENCES}

[1] National Grid, "Future Energy Scenarios," National Grid, Warwick, 2017.

[2] C. Rahmann, J. Jara and M. B. C. Salles, "Effects of Inertia Emulation in modern Wind Parks on Isolated Power Systems," in IEEE Power \& Energy Society General Meeting, Denver, 2015.

[3] F. M. GONZALEZ-LONGATT, "Impact of emulated inertia from wind power on under-frequency protection schemes of future power systems," Journal of Modern Power Systems and Clean Energy, vol. 4, no. 2, pp. 211-218, 2015.

[4] A. Junyent-Ferré, Y. Pipelzadeh and T. C. Green, "Blending HVDC-Link Energy Storage and Offshore Wind Turbine Inertia for Fast Frequency Response," IEEE TRANSACTIONS ON SUSTAINABLE ENERGY, vol. 6, no. 3, pp. 1059-1066, 2015. 
[5] H.-P. Beck and R. Hesse, "Virtual synchronous machine," in 9th International Conference on Electrical Power Quality and Utilisation, Barcelona, Spain, 2007.

[6] T. Qoria, F. Gruson, F. Colas, X. Guillaud, M.-S. Debry and T. Prevost, "Tuning of Cascaded Controllers for Robust GridForming Voltage Source Converter," in 2018 Power Systems Computation Conference (PSCC), Dublin, 2018.

[7] Brunel University London, "Investigation \& Modeling of Fast Frequency Phenomena, WP1 - Data Gathering, Visualisation and Investigation,” Brunel University London, London, December 2018.

[8] Energy Networks Association, "Loss of mains protection settings for all small generators, stakeholder workshop," Energy Networks Association, London, 2018.

[9] National Grid ESO, "Monthly Balancing Services Summary 2019/20 January 2020," [Online]. Available: www.nationalgrideso.com/document/165056/download .

[10] National Grid Electricity System Operator, "National Grid ESO outline new approach to stability services in significant step forwards towards a zero-carbon electricity system," [Online]. Available: www.nationalgrideso.com/media/national-grid-esooutline-new-approach-stability-services-significant-step-forwardstowards.

[11] "Engineering Recommendation G59, Issue 3 Amendment 7, Recommendations for the Connection of Generating Plant to the Distribution Systems of Licensed Distribution Network Operators," Energy Networks Association, London, 01 September 2019.

[12] Energy Networks Association, "Accelerated loss of mains change programme," [Online]. Available: www.energynetworks.org/electricity/engineering/accelerated-lossof-mains-change-programme.html ].

[13] National Grid Electricity System Operator, "Technical Report on the events of 9 August 2019," National Grid Electricity System Operator, Warwick, 2019.

[14] P. Imris, G. Taylor and I. Pisica, "Investigation \& Modelling of Fast Frequency Phenomena - WP1," Brunel University London, London, 2019.

[15] P. Imris, M. Bradley, G. Taylor, M. Gordon and Y. Li, "Enhanced visualisation of Fast Frequency Phenomena as exhibited in the GB transmission system," in The 54th International Universities Power Engineering Conference, Bucharest, Romania, 2019.

[16] P. M. Ashton, C. S. Saunders, G. A. Taylor, A. M. Carter and M. E. Bradley, "Inertia Estimation of the GB Power System Using Synchrophasor Measurements," IEEE Transactions on Power Systems, vol. 30, no. 2.

[17] SP Energy Networks, "Visualisation of Real Time System Dynamics using Enhanced Monitoring (VISOR) - Close Down Report," SP Energy Networks, Blantyre, 2018, Available: www.spenergynetworks.co.uk/pages/visor.aspx.
[18] National Grid, "Electricity Ten Year Statement 2018," National Grid plc, Warwick, 2018.

[19] D. Rostom, "Creation of a Reduced Model for the GB network," National Grid Draft Report, Warwick, 2013.

[20] P. Imris, M. Bradley, G. Taylor and Y. Li, "A Novel Hardware-Inthe-Loop Approach to Investigate the Impact of Low System Inertia on RoCoF Relay Settings," Submitted to IET Generation, Transmission \& Distribution, May 2020.

[21] V. Jalili-Marandi, F. J.Ayres, E.Ghahremani, J. Bélanger and V. Lapointe, "A real-time dynamic simulation tool for transmission and distribution power systems," in IEEE Power Energy Soc. Gen. Meeting, 2013.

[22] OPAL-RT, "ePHASORSIM User Guide," [Online]. Available: www.opal-rt.com/systems-ephasorsim/. [Accessed 1910 2019].

[23] B. A. Osbouei, G. A. Taylor, O. Bronckart, J. Maricq and M. Bradley, "Impact of Inertia Distribution on Power System Stability and Operation," in 13th IEEE PowerTech 2019 conference, Milan, 2019. 